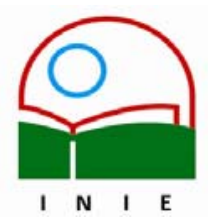

Universidad de Costa Rica

Facultad de Educación

Instituto de Investigación en Educación

ACTUALIDADES INVESTIGATIVAS EN EDUCACION

\title{
DESARROLLO DE LA LECTURA MEDIANTE ESTRATEGIAS INTEGRADORAS
}

Maira Solé

Resumen: La lectura y la escritura son procesos que cada día ameritan nuevos cambios y transformaciones. La propuesta de un Proyecto Pedagógico Integrador, (Fraca 2003) desarrollado con éxito en algunas instituciones venezolanas, se perfila como una alternativa significativa para el desarrollo de estos elementos. La idea o núcleo central es la integración de las diferentes asignaturas curriculares y lograr una globalización partiendo de sus objetivos y contenidos programáticos. El eje pedagógico integrador le permite al docente, evidenciar con mayor prontitud los resultados mediante actividades prácticas de lectura y escritura. Así mismo combina elementos claves del aprendizaje ausbeliano: información previa, información nueva y construcción de la información definitiva o integrada. La puesta en ejecución de las estrategias integradoras, en esta ocasión por maestros en formación (UNEG), a diferentes niños de escuelas del Estado Bolívar (Venezuela), certificando cómo la lectura y la escritura pueden tener un espacio ideal y significativo en la instrucción actual. Solo se necesita la intención, creatividad, dinamismo e ingenio.

Palabras clave: ESTRATEGIA INTEGRADORA/EJE PEDAGÓGICO/PROYECTO INTEGRADOR/ LECTURA/

\begin{abstract}
The reading and the writing plows processes that every day they require new changes and transformations. The proposal of an Integrative Pedagogic Project, (Fraca 2003) developed with success in some Venezuelan institutions; it is profiled like a significant alternative for the development of these elements. The idea or central nucleus is the integration of the different curricular subjects and to achieve a globalization leaving of its objectives and programmatic contents. The integrative pedagogic axis allows to the educational one, to evidence with more readiness the results by means of practical activities of reading and it notarizes. Likewise it combines key elements of the learning ausbeliano: previous information, new information and construction of the definitive or integrated information. The operation of the integrative strategies, in this occasion for teachers in formation (UNEG), to different children of schools of the State Bolivar (Venezuela), certifying how the reading and the writing dog have an ideal and significant space in the current instruction. Alone it is needed the intention, creativity, dynamism and genius.
\end{abstract}

Key words: INTEGRATIVE STRATEGY/ PEDAGOGIC AXIS/ INTEGRATIVE PROJECT/ READING/

\section{1.- Introducción}

El trabajo que a continuación presento sobre estrategias integradoras, tiene como objetivo primordial el perfeccionamiento de la lengua oral y escrita a través de prácticas didácticas de índole psicosociolingüístico. Este elemento es quien brinda a la presente temática un estilo diferente en lo que estrategias de lectoescritura se refiere. Su carácter globalizador, constituye el centro de atención de este trabajo. Aparte de la creatividad, sencillez y flexibilidad que identifica de manera particular a cada aplicación didáctica.

\footnotetext{
${ }^{1}$ Magíster en Planificación y Evaluación Educativa (INFORHUM). Se desempeña en el área de Lengua en la Universidad Experimental de Guayana, sede Ciudad Bolívar (UNEG-Bolívar, Venezuela) en asignaturas como: Didáctica de la Lengua y la Literatura, Lengua I y II, Literatura Infantil, Lectoescritura entre otras. Docente en la Especialidad de Lingüística y Literatura. Egresada del Instituto Pedagógico de Maracay (UPELIPMAR). Correo electrónico: castillosole@cantv.net
}

Artículo recibido: 17 de marzo, 2005

Aprobado: 27 de iunio, 2005 
Muchas de las estrategias de lectura y escritura existentes plantean un abordaje específico, ya sea para la lectura o para la escritura e incluso inclinadas a un área particular, lo cual imposibilita al maestro extender su aplicación a otras áreas y otros momentos instruccionales. De esta preocupación surgen las estrategias integradoras; las cuales vienen a responder interrogantes de los docentes y a ejecutar acciones efectivas en el campo oral y escrito del alumno, donde ambos estén concientes del uso efectivo de su lengua materna.

La autora de las estrategias integradoras Lucía Fraca (2003) fundamenta su Proyecto Pedagógico Integrador (PEI) en el modelo de aprendizaje de Vigotsky (1973) y el aprendizaje significativo de Ausubel (1976). De esta forma las estrategias se sustentan en un aprendizaje por mediación que considera la información previa del alumno para la transmisión de la nueva y poder llegar entonces a la transferencia integral.

Es necesario establecer los aspectos teóricos de Ausubel y Vigotsky que Fraca considera en el desarrollo del PEI. En cuanto al modelo de Ausubel se caracteriza fundamentalmente en la transmisión que el maestro desarrolla al momento de presentar la información a los aprendices; por lo tanto existe una estrecha relación entre la estrategia integradora y el estudiante, específicamente a nivel cognoscitivo.

Una concepción del aprendizaje en estos parámetros, supera la rutina escolar, la memorización tradicional en las aulas y da paso al aprendizaje integrador, comprensivo y autónomo. En cuanto a esto volveremos más adelante ya que son la columna de las estrategias integradoras.

La práctica docente demuestra que el punto de inicio es el conocimiento que el niño tiene o conoce, respecto a lo que pretende aprender. En tal sentido la propuesta de Fraca guarda una estrecha armonía con la teoría de Ausubel. Debido al interés en los procesos de aprendizaje relacionados con la internalización de los conceptos científicos a partir de aspectos previamente formados por el aprendiz en su entorno sociofamiliar. Las estrategias entonces se encaminan a la organización del conocimiento, se reestructuran gracias a la interacción de lo previo y lo nuevo. Esta acción da como resultado un Eje Pedagógico Integrador (EPI) sustentado en un aprendizaje activo y significativo. 
Los aportes de Vigotsky (1973) considerados en el desarrollo de la pedagogía integradora son los referentes al lenguaje como instrumento de intercambio sociocultural del ser humano. En este caso el alumno posee dos niveles de desarrollo: el actual y el potencial; este último referido a la solución de problemas a lo que recibe la ayuda de un experto, llámese maestro o su propio compañero de estudios. La distancia que existe entre estos dos niveles el autor la denominó Zona de Desarrollo Próximo (ZDP).

En la medida que en salón de clases se convierte en un centro de interacción en esa medida el discente construye su ZDP.

El propósito de Fraca al utilizar ambos fundamentos es darle el carácter integrador a las estrategias y por supuesto a la lengua materna oral y escrita. Un uso realmente efectivo, propiciando situaciones comunicativas de diversa índole: elaboración de murales, periódicos, guiones de títeres, trípticos informativos, rondas de cuentos, que son parte de las estrategias propuestas por la autora, la cuales en líneas generales abordan las diferentes dimensiones de la lengua. Esto permitirá que el proceso de lectura se dinamice y salga de la monotonía en que se encuentra.

El alumno está a la expectativa de novedades de lectura y de escritura en el aula; que el maestro deje de lado la recarga de asignaciones sin sentido y repetitivas o lecturas sin sentido. Que pueda llevar ofrecimientos diferentes a cada encuentro escolar, que le brinde la oportunidad de proponer e intervenir, en fin de ser escuchado. Esto es uno de los aspectos más atrayentes del estudio: presentar una herramienta significativa para abordar la lectura y la escritura dentro y fuera del aula.

Se pretende que este diseño pueda ser enriquecido con cada experiencia docente, la cual puede seguir aportando a cada estrategia un abordaje distinto; pues uno de los elementos que se manifiesta en el PEI es la flexibilidad y adaptabilidad de estas herramientas. La práctica efectuada por estudiantes universitarios en Educación Integral (UNEG, 2004-II) demostró la efectividad de las estrategias integradoras de aula en Educación Básica.

\section{2.- Puntos de encuentro.}

La lectura y sus estrategias han sido objeto en los últimos años de grandes e interesantes investigaciones: Solé 1992, Elosúa y García 1993, Ferreiro y Gómez (1997); Kintsch 1998, 
Cassany 1999; Teberosky 1992; entre otros. Cada uno ofreciendo aportes en pro del perfeccionamiento de la lectoescritura.

Esta ardua tarea académica posee diversidad de factores implicados, pero de toda la teoría existente, encontramos puntos en común como por ejemplo:

1. Una estrategia de aprendizaje debe contribuir a que el alumno optimice sus procesos de metacomprensión.

2. Existen estrategias para cualquier momento instruccional: antes, durante y después. (Solé I,1992)

3. Lo más importante no es que los alumnos posean un amplio repertorio de estrategias, sino que sepan utilizarlo eficazmente según un propósito determinado.

4. Determinar contexto, propósito, texto y/o material instruccional son piezas que permiten armar nuestro gran rompecabezas, cuya forma definitiva no será tan sólo imagen, como es lo habitual, sino que podrá ser audible (oralidad) y comprobable a través de un producto (escritura). Esto será realmente el éxito estratégico.

\section{3.- Estrategias Integradoras.}

Atendiendo a las múltiples necesidades del Currículo Básico Nacional (CBN) y a las demandas educativas a nivel general que el sistema educativo venezolano enfrenta día a día, la profesora Lucía Fraca (2003) mediante una propuesta bien sustanciada propone la creación de una pedagogía Integradora; cuyo énfasis fundamental se halla en las estrategias, lograr la integralidad en las diferentes áreas de aprendizaje es el foco de interés de tan significativa propuesta.

Las estrategias integradoras de aula están inmersas dentro del Eje Pedagógico Integrador (EPI), el cual a su vez forma parte de lo que la autora denomina Proyecto Educativo Integrador (PEI) ( Fig. 1) 


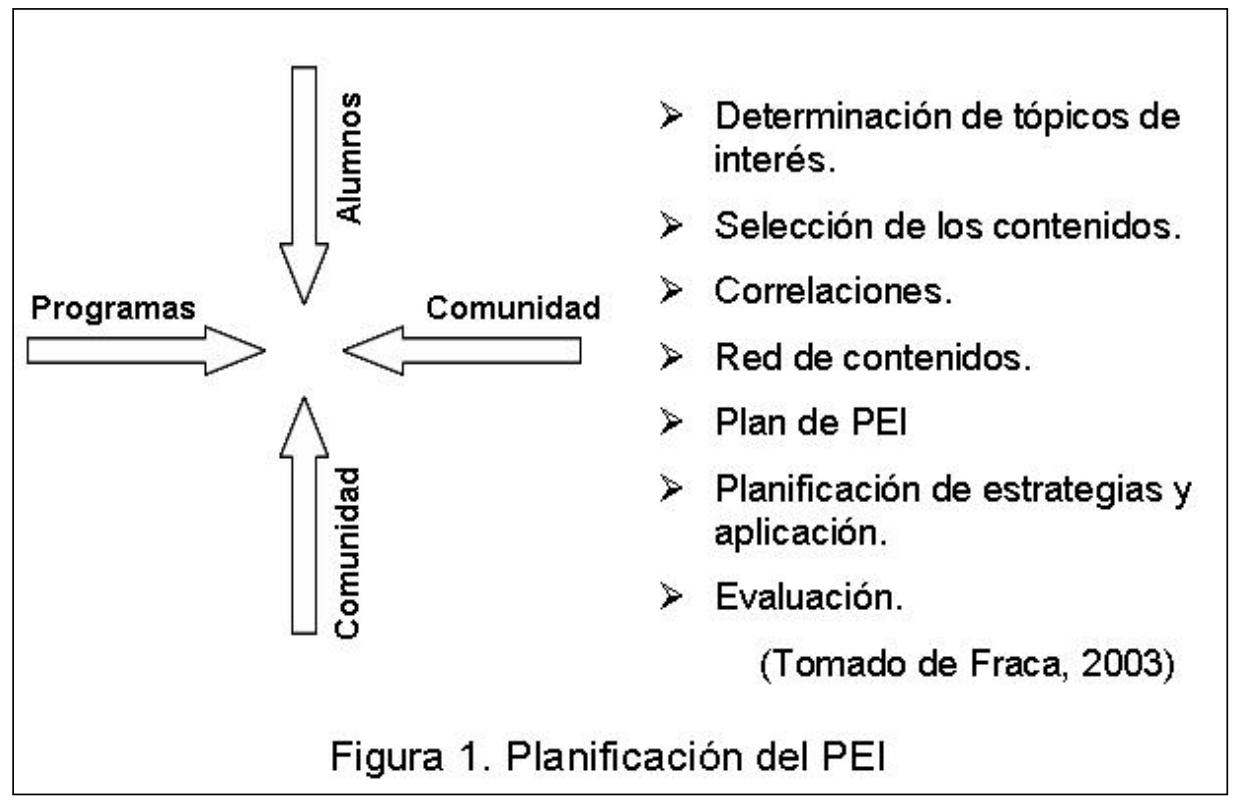

EI PEI involucra a todos los agentes educativos a través de la participación en actividades específicas ya sean de diseño, planificación o ejecución. Esto le brinda un carácter innovador a la lectura, saliendo de la monotonía del proceso cotidiano del aula de clase, en donde el niño escasamente lee unas líneas al maestro, con poca o ninguna interpretación pues en la mayoría de los casos suele ser mecánica y repetitiva y el que participa en cada proceso es el maestro.

Es la pedagogía integradora la que permite un aprendizaje dinámico, globalizador y cíclico, donde las estrategias cognoscitivas le permiten al alumno comprender sus procesos de reflexión y análisis, de un texto específicamente (metacognición, metacomprensión) de una forma responsable, donde él mismo puede comprobar sus avances ya sea en su comprensión de texto o en la producción escrita.

Las estrategias de lectura y escritura llevan intrínsecamente una serie de misiones, las cuales se desarrollan en el tiempo y en el espacio en forma constante y progresiva. En tal sentido no habrá éxito estratégico sin una debida ejercitación prolongada de esta. Díaz y Hernández (2003, p. 304) refieren que el aprendizaje de estrategias orientadas a la lectura deben reunir las siguientes exigencias mínimas:

- Conocimiento de la estrategia.

- Conocimiento detallado de su ejecución. 
- Conocimiento sobre su funcionalidad y ejecución.

- Saber en qué contextos y en qué situaciones de lectura o no recomendable su uso.

- Su autorregulación es progresiva.

- Su flexibilidad.

Las estrategias deben practicarse, hasta que llega el momento que el alumno comienza a realizarlas por su propia cuenta. Además son planificadas tomando como referencia el EPI: información previa, nuevo conocimiento y transferencia de lo aprendido. Para lograr que sean efectivas el proyecto se ha cimentado en las teorías de Ausubel (1986), en lo relacionado al aprendizaje significativo y en la de Vigotsky: zona de desarrollo potencial. Ambos estudios fortalecen los procesos psicosociolingüísticos, dando coherencia al empleo de la lengua oral y escrita.

Una visión holística del proyecto y sus estrategias, se convierten en un conjunto de acciones mas específicas, que permiten obtener un producto inmediato, quizás sea por lo sintético de los pasos a seguir, haciendo que el maestro se emocione al igual que sus alumnos. Aunque no es el tema de interés en este momento, es necesario saber que los proyectos integradores poseen tres fases: selección de los contenidos, selección de los proyectos y elaboración de la red de contenidos; evitando repeticiones, saltos o regresiones en la planificación y no prolongar en el tiempo la estrategia para ver su resultado.

\subsection{Identificación de una Estrategia Integradora}

Las estrategias integradoras tienen la particularidad de favorecer el desarrollo de las competencias lingüísticas, demarcadas a través de los cuatro ejes del lenguaje: hablarescuchar; leer-escribir. Por consiguiente se considera como marca significativa, el hecho de trabajar con el conocimiento previo del alumno; haciéndole sentir al niño que su vivencia es importante y válida, permite que la estrategia tenga mayor sentido dentro de la instrucción.

Otro factor que contribuye a la identificación de la estrategia integradora es la indagación; cuánto conoce el discente sobre el contenido programático (nueva información); pues de ello va a depender que la estrategia se cambie o modifique. En último lugar está la integración o globalización entre el conocimiento previo y el nuevo. Son pues los postulados de Ausubel (1976) y los de Vigotsky (1973) los que logran establecer la conexión entre la integración estratégica propuesta por Fraca, la información que el alumno conoce y la nueva que ha de 
recibir y su respectiva correspondencia con el pensamiento y el lenguaje. Es en esta medida que alcanzaremos un individuo social y lingüísticamente competente (Fig.2).

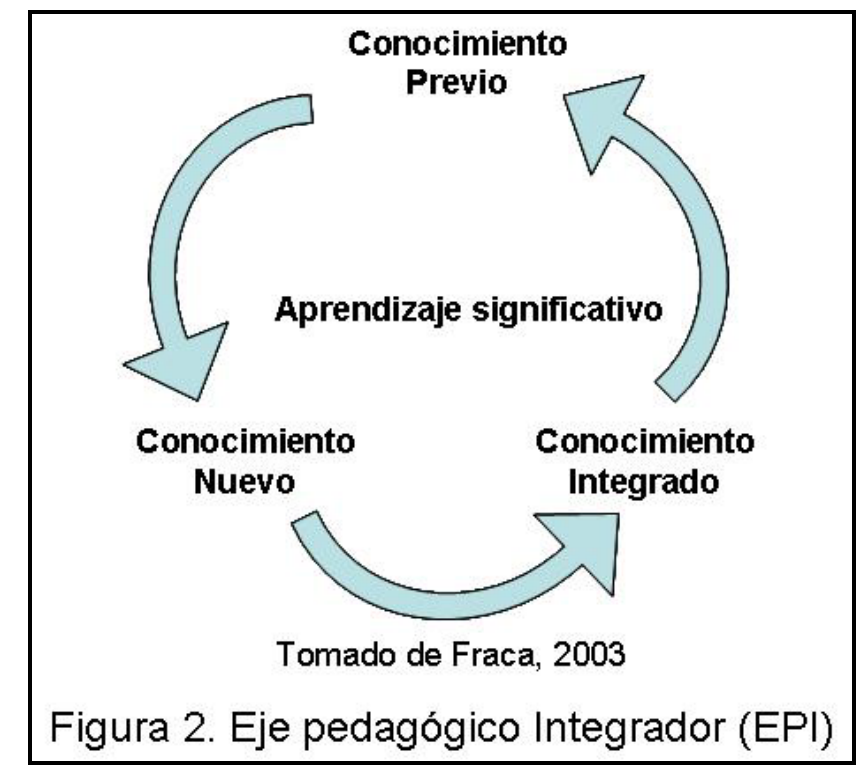

Una estrategia integradora, además de lo antes mencionado, tiene además rasgos que la definen como tal, los cuales le permitir al docente discernir el tipo de estrategia a ejecutar entre ellas se encuentran:

- Naturalidad: una estrategia didáctica integral debe llevar una intención lingüística de por medio, pues va orientada a un público que descubre y aprende de sí mismo, mediante la interacción con su realidad, con su entorno, el cual no se puede dejar a un lado, pues es su vida. La relación con sus múltiples situaciones le permiten al alumno confrontar sus ideas, sus pensamientos y emociones, al ver como sus compañeros resuelven los problemas que plantea el lenguaje, al obtener información de los demás, así son y así aprenden. (Álvarez, 2001, p.11).

En forma similar ocurre con todo lo que se desarrolla en el aula: libros, revistas, cuentos, folletos, periódicos deben estar enmarcados dentro de un contexto socialmente definido, para que pueda ser significativo para el alumno.

- Significativas: para lograr el mayor beneficio de las estrategias, es necesario que tengan una razón de ser dentro de ese contexto. En oportunidades el docente se deja llevar por su propio interés o motivación ante determinado material, pero cabe preguntarse, ¿será significativa esa información para el niño? Para evitar los desaciertos, la actividad escolar debe estar enmarcada en una correspondencia: 
demandas e intereses del aprendiz. Si no es relevante para él entonces no producirá el efecto deseado.

- Motivantes: La emoción y el movimiento, la pasión por lograrlo es fundamental. Si es significativa y natural, pero carece del acompañamiento docente, es probable que decline su efectividad y por consiguiente su matiz globalizador.

- Creativas: así como la mediación y el acompañamiento deben ser constantes. El promover e impulsar a la creatividad ha de ser igual, cómo hacerlo: ayudándolos a descubrir nuevas formas de hacer las cosas, estando cerca de ellos, hacerlos pensar y reflexionar a ir más allá del conocimiento a intentar a innovar.

- Dinámicas: todo es comunicación, todo es lenguaje, así Álvarez (2001) expresa que esa energía vital de cada niño: sus expresiones de asombro, alegría, enojo, sus gestos nos hablan de sus necesidades. Todo un lenguaje, una relación armónica de lo que somos. Eso es la estrategia integradora un todo indisoluble que llamamos Lenguaje: hablar y escuchar, leer y escribir.

\subsection{Contenido de la Estrategia Integradora}

La estrategia integradora está constituida por los elementos del EPI (Fig.2) El conocimiento previo, el conocimiento nuevo y el conocimiento integrado. Esto significa un aprendizaje activo, cíclico y globalizado.

En relación al conocimiento previo le va a permitir al maestro diagnosticar qué realmente, de lo cotidiano, conoce el alumno. Cuáles elementos pueden dar indicios de ubicación actual y próxima. Una vez que se ha delimitado este primer conocimiento se incorpora la información nueva, donde el docente implementa formas para lograrlo: preguntas y respuestas, lluvia de ideas entre otras. Por último se encuentra la integralidad. La aplicación de ese conocimiento en diversas situaciones, la transferencia de la información por medio de estrategias. Una vez que hemos recorrido y asimilado el EPI, podemos seleccionar con precisión cuál de las estrategias integradoras es la que mejor se adapta al contenido a desarrollar.

La estrategia integradora está conformada por cinco componentes:

\section{Título de la estrategia}

2. Propósito: por qué y para qué se va a aplicar una determinada estrategia.

3. Nivel de aplicación: indica el grado o nivel de educación básica donde se ejecutará la estrategia.

4. Bloques de contenidos: de acuerdo a lo que establece el currículo básico nacional se consideran los bloques siguientes para el área de Lengua y Literatura: EI 
Intercambio oral, ¡A Leer y a Escribir!, Reflexiones de la lengua y la literatura: el mundo de la imaginación

5. Descripción: son las actividades que se efectuarán para concretar la estrategia.

\subsection{1era Muestra de Estrategias Integradoras de Aula}

De las treinta (30) estrategias que conforman el proyecto de Fraca, los alumnos de Educación Integral de la Universidad Nacional Experimental de Guayana (UNEG), seleccionaron 22 de ellas las cuales aplicaron en algunas instituciones educativas de la localidad, obteniendo óptimos resultados.

En primer término seleccionaron la estrategia, luego determinaron el nivel (grado) donde deseaban aplicarla y el bloque de contenido, a pesar de que las estrategias ya están prediseñadas, la mayoría de los alumnos cambió el nivel y variando los diferentes subniveles del área de lengua, los que más fueron trabajados fueron el intercambio oral y el mundo de la imaginación, algunos argumentaron que a los niños les divierte el hecho de crear, relatar sus vivencias e imaginarse otras. De igual forma se evidenció que el hecho de seguir instrucciones es decir la descripción de la estrategia por parte del maestro (estudiante UNEG) fue clave para el éxito de la actividad.

En relación a los recursos resultaron accesibles, en algunas sólo se requería dinamismo y compenetración con el grupo de niños para llegar a cumplir el propósito planteado. Se comprobó también que esta forma le permite al docente comprobar, de forma casi inmediata, la asimilación de los contenidos por parte del alumnado.

Los alumnos de Educación Integral presentaron esta experiencia como trabajo final de la cátedra: Electiva de Lengua (VIII semestre), en una actividad dirigida a toda la comunidad unegista cuyo titulo fue 1era Muestra de Estrategias Integradoras de Aula.

Una de las estrategias que deseo compartir se titula "Las Cuevas"; a continuación se describe su aplicación (Fraca, 2003, p. 128-129):

\section{Propósito:}

Esta estrategia permite la discriminación de sonidos naturales característicos del contexto o ambiente que rodea al alumno. De igual modo favorece la atención y la selección de 
aspectos relativos ala vida en sociedad. A parte de "Las Cuevas" existen 29 estrategias adicionales orientadas al desarrollo de los diferentes ejes de la lengua (hablar-escuchar; leer-escribir) tanto en forma individual como combinada.

Nivel de Aplicación: Preescolar y primer grado.

Bloque de Contenido: El Intercambio Oral

\section{Descripción:}

A un niño, protagonista del juego, se le separa del resto del grupo; éste se distribuye en siete cuevas:

- Cueva de la risa

- Cueva de la tos

- Cueva del estornudo

- Cueva del ronquido

- Cueva del llanto

- Cueva del silbido

- Cueva del silencio

El protagonista (niño/a), simulando tener sueño, busca cobijo en una cueva.

Se acerca a la primera cueva y apenas recostado comienza una risa estrepitosa. Uno de sus compañeros ríe constantemente impidiendo que el otro duerma en paz. Por medio de la risa, aunque fingida, el niño puede demostrar como un hecho tan natural como la risa en ocasiones puede perturbar si no es el lugar y momento indicados. Se aprecia que el resto de los compañeros que participan, están aguantando su deseo de reír, otros por el contrario hacen silencio en espera de la cueva siguiente.

Ante la imposibilidad de conciliar el sueño, acude a la segunda, pero se repite la escena y una tos incesante no lo deja dormir. Esta cueva resulta muy interesante pues los niños asocian que la persona está enferma y de allí su tos. Al igual que la anterior evalúan como una tos llega a convertirse en un elemento perturbador a la hora del descanso. Se observa además que el grupo siempre está a la expectativa y demuestran interés y asombro ante las escenas que se van desarrollando. Los niños hacen más silencio cuando el niño llega a la cueva, es una especie de curiosidad en sus mentes, la cual anhela ser despejada con prontitud. 
Aturdido llega a la tercera cueva pero un estornudo alérgico le impide cumplir su tan anhelado objetivo: dormir. El estornudo como síntoma de alergia o enfermedad le impide al pequeño descansar y se ve obligado a abandonar el lugar. Algunos de los niños dice con voz fuerte ¡déjalo dormir!; lo cual hace suponer que ya disciernen que para poder descansar hay que hacer silencio. Se aprecia otra constante en esta fase: los diálogos en voz baja entre los niños que observan la simulación. Esto como indicio de su participación y espontaneidad ante la presentación.

La cuarta cueva sorprende al niño ya que unos ronquidos fuertes y prolongados le instan a abandonar el lugar. Algunos dicen expresiones como: hace como mi papá o hay que feo hace. Esto demuestra la asociación que el niño hace ante un sonido conocido.

Llega así a la quinta cueva, nada agradable pues un llanto triste y melancólico es quien le recibe. La situación comienza a entristecer a nuestro amigo; quien aún no pierde las esperanzas de descansar. Emociones diferentes afloran si comparamos esta cueva con la primera (risa). Es casi imposible que el pequeño relaciones el llanto con la nostalgia, dolor o melancolía. El niño que dramatiza camina de un lado a otro y desea pronto abandonar ese sitio.

Cansado ya, se aproxima a la sexta cueva, donde es recibido por un silbido muy largo, prácticamente se encuentra extenuado y sin fuerzas. Este sonido no le gusta al pequeño que simula las acciones. Está inquieto y con deseos de salir de allí. Los otros participantes gritaron un poco diciendo: uhhh que miedo; lo que puso más nervioso al dramatizador de escenas.

Al llegar a la séptima cueva observa que no hay ruido alguno es lógicamente ¡La cueva del silencio! Tan profundo era su sueño que ni las llamadas de sus compañeros lograban despertarlo. Solamente lo consiguió cuando todas las cuevas al unísono emitieron sus propios sonidos. (Esta estrategia fue tomada de M Paz y M. Lebrero, 1999).

A lo largo del desarrollo de Las Cuevas se pudo apreciar la correspondencia estrecha entre los planteamientos de Ausubel: el niño que intenta dormir recibe una información verbal de parte de sus compañeros (risa, silbido, ronquidos, etc.) y de inmediato vincula con situaciones similares, dónde ha escuchado ese sonido, a quién se lo ha oído, por qué no 
puede descansar, son entre otras las interrogantes que se plantean los pequeños, las cuales da respuesta a medida que establece conexiones entre lo nuevo y lo que ya conoce (1986, p. 45).

De igual manera acontece con los postulados de Vigotsky. Al momento que la docente explica las instrucciones y prepara al grupo para que participe de la estrategia se evidencia su desarrollo actual, en la medida que comienza la interacción con sus compañeros va rumbo a su desarrollo potencial. Se evidenció además que a pesar de ser niños de preescolar y primer grado la Zona de Desarrollo Próximo (ZDP) es mínima, lo cual demuestra que estos grupos son activos y participativos y sus relaciones interactivas emprendedoras.

\section{Conclusiones}

Las estrategias integradoras le permiten al niño aprovechar al máximo su potencial lingüístico-cognitivo mediante prácticas pedagógicas significativas. Los procesos de lectoescritura se enriquecen mediante la globalización estratégica expuesta por Fraca.

Una pedagogía de aula bajo una concepción integradora, pronostica resultados satisfactorios a todo nivel. Estas estrategias han venido a satisfacer la demanda de muchos docentes, que con el pasar de los años exigían al sistema educativo actualizaciones acordes a la realidad circundante. La autora pone al alcance del maestro una herramienta de gran provecho académico, donde la lengua es el elemento principal, específicamente la interacción, ya que será la que conlleve al aprendizaje significativo del alumno y logre la interrelación entre sus niveles de desarrollo actual y potencial.

La planificación y ejecución del Proyecto Educativo Integrador (PEI), propuesto por Lucía Fraca, contribuye en cierta medida al fortalecimiento de los Proyectos Pedagógicos de Aula, diferenciándose por su Eje Pedagógico Integrador (EPI), el cual promueve la interacción motivadora entre el docente y el alumno.

Son las Estrategias Didácticas Integradoras las que facilitarán la mediación para la adquisición de competencias cognoscitivas, lingüísticas y comunicativas por parte del alumno a través del modelo integrador. Su aplicación consiste en un método versátil, adaptándose a las necesidades de cada grupo y a la realidad de cada institución. El docente puede entonces integrar estrategias, de las 30 que se proponen. Todo va a depender de la 
interacción escolar creativa, natural y significativa que el maestro promueva en el aula. la escuela se convierte en un espacio de intercambio y afianzamiento de los valores individuales y de la identidad nacional y cultural. Una muestra de ello lo constituye el trabajo realizado por estudiantes de pregrado en Educación Integral de la Universidad Experimental de Guayana (UNEG-Ciudad Bolívar, Venezuela). El cual se expone a continuación como una evidencia más de lo que las estrategias integradoras permiten en el aula.

La 1era Muestra de Estrategias Integradoras de Aula sirvió para exponer veinte (20) de las treinta estrategias propuestas por Fraca. En ella los alumnos seleccionaron una etapa y un grado de Educación Básica y aplicaron la estrategia asignada en escuelas locales, obteniendo óptimos resultados, entre los cuales tenemos los siguientes:

- Asociación por parte del niño en lo relacionado al nombre de la estrategia y que ella representa.

- Seguimiento de instrucciones garantizando el éxito en la aplicación.

- Participación espontánea de los alumnos en la ejecución de la estrategia, en el caso de Las Cuevas en forma oral, concentrándose esta en los ejes expresar-escuchar.

- Esta primera muestra de estrategias integradoras de aula ha dado origen a otras investigaciones a corto y mediano plazo, las cuales se han comenzado a realizar a través de la Coordinación de Proyectos de Investigación y Desarrollo (PID) en la UNEG-Bolívar

Esto demuestra en parte la efectividad de las estrategias integradoras y por ende al desarrollo de Proyectos Educativos Integradores (PEI), de acuerdo a la experiencia y los resultados obtenidos hasta la fecha. Queda pues un camino estratégico por andar donde maestro, alumno y comunidad se integran para alcanzar un aprendizaje de calidad y significativo para todos.

Las práctica pedagógica debe estar en estrecha vinculación a las estrategias de lectura capaces de captar la atención y el interés del discente; eliminar aquellas actividades rutinarias y pasivas donde el alumno lo que hace es incrementar su rechazo hacia la lectura, este es realmente el acompañamiento académico que el docente ofrece a sus aprendices. 
Las estrategias integradoras para la enseñanza y aprendizaje de la lectura y escritura son de gran importancia, puesto que la aprehensión de los contenidos a desarrollar en una clase, aparte de los valores conocidos, deben asegurar un proceso interactivo, donde los actores puedan participar y establecer un diálogo permanente. Un lugar para los cambios personales, sociales y culturales. Estos procedimientos estratégicos de enseñanza deben ser utilizados en forma flexible con el propósito de promover la mayor cantidad y calidad de aprendizaje significativo en los alumnos. Debe hacerse un uso inteligente, adaptativo e intencional de ellas, con la finalidad de prestar la ayuda pedagógica asertiva.

Una pedagogía que se desarrolla según los lineamientos expuestos le permitirá al docente reforzar su rol de mediador y establecer puntos de encuentro entre la información conocida y la nueva, aparte de establecer una integralidad de los procesos de lectura y escritura, mediante actividades dinámicas, sencillas y productivas.

Deseo expresar mi agradecimiento de manera muy especial a los alumnos del VIII semestre (2004-II) de la carrera de Educación Integral (UNEG- Bolívar), quienes formatearon toda la información y diseñaron esta 1era Muestra de Estrategias Integradoras de Aula. Esta acción tuvo lugar en el marco de la Antesala de una actividad mayor denominada Expodidáctica, la misma se desarrolla bajo el apoyo del Proyecto de Carrera de Educación Integral y su finalidad es la exposición significativa de estrategias de enseñanza y aprendizaje. La misma tiene como lema para este año: "Hacia la calidad de los procesos productivos de la academia".

Finalizo haciendo un llamado al maestro del siglo XXI, en relación a que en ocasiones nos anclamos en nuestras filosofías y formas únicas de lograr un producto de calidad en nuestros alumnos y culpamos a todo y a todos; hoy hemos visto que con elementos fáciles de aplicar y pocos recursos el alumno puede alcanzar el éxito, demostrables a través de un empleo efectivo de la lengua oral y escrita. 


\section{Referencias}

Álvarez, M. (2001). Pequeños lectores, escritores y poetas. Juegos de lenguaje para niños de 2 a 6 años. México: Noriega.

Ausubel, D. (1976). Psicología educativa. México: Trillas.

Ausubel. D, Novak. J y Hanesian. H. (1986). Psicología Educativa. Un punto de vista cognoscitivo. México: Trillas

Cassany, Daniel. (1990). Enfoques didácticos para la enseñanza de la lengua escrita. Comunicación, lengua y educación, 6, 68-80.

Díaz, F y Hernández G. (2003). Estrategias Docentes para un Aprendizaje Significativo. Una interpretación constructivista. México: Mc Graw-Hill.

Elosúa, M y García, E. (1993). Estrategias para enseñar y aprender a pensar. Madrid: IEPS-Narcea.

Ferreiro, E y Gómez, M. (Comps). (1997). Nuevas perspectivas sobre los proceso de lectura y escritura. México: Siglo XXI.

Fraca, L. (2001). La comprensión de materiales escritos en la escuela. Candidus, 17, 2025.

(2002). Una didáctica estratégica para la lengua escrita: aplicaciones para el Currículo Básico Nacional. Lingua Americana, 5, 1-31.

. (2004). Pedagogía Integradora en el Aula. Caracas: El Nacional.

Kintsch, W. (1998). Comprensión: un paradigma cognitivo. Cambribge: Cambribge University y Press.

Paz, M y Lebrero, M. (1999). Cómo y cuándo enseñar a leer y escribir. Madrid: Síntesis.

Solé, I. (1992). Estrategias de Lectura. Barcelona: Graó-ICE.

Teberosky, A. (1992). Leer para enseñar a escribir. Cuadernos de pedagogía, 216, 22-24.

Vigostky, L. (1973). Pensamiento y lenguaje. Buenos Aires: La Pléyade. 